\title{
The Number of Lymph Nodes That Can Be Added to an Abdominal Flap for Breast Reconstruction: Review of Enhanced MDCT Images
}

\author{
Kenta Tanakura, MD*; Akiko Matsumoto, MD; Yukiko Kuramoto, MD; Masayuki Sawaizumi, MD \\ The Cancer Institute Hospital, Japanese Foundation for Cancer Research, Koto, Tokyo, Japan
}

\begin{abstract}
Objectives: Vascularized lymph nodes transfer (VLNT) has been noticed as an effective treatment for lymphedema. Several sites of lymph nodes have been reported as donor sites, and the inguinal nodes are one of the major donor sites for upper extremity lymphedema. These nodes enable to perform VLNT and breast reconstruction by transferring an abdominal flap containing inguinal nodes. Our study aimed to recognize how many nodes could be added to the abdominal flap.

Methods: We reviewed 48 cases and 96 sides multi detector computed tomography (MDCT) images, which were taken from 2011 to 2014 , to prepare for deep inferior epigastric perforator (DIEP) flap. All the cases were females. We counted the number of inguinal lymph nodes. To avoid the donor site mobility such as newly caused lymphedema, the superficial inguinal nodes sited superior to the junction of femoral vein and greater saphenous vein are appropriate.

Results: The number of lymph nodes ranged between 0 and 10, with 3 in 25 sides (26\%) and 4 in 18 sides (19\%). The average was 3.67 for single side. No significant difference existed between left and right sides.

Conclusion: The lymph nodes-added abdominal flap transfer is a promising procedure, which enables breast reconstruction and lymphedema treatment at the same time. Inguinal nodes are fed by superficial circumflex iliac artery (SCIA), superficial inferior epigastric artery (SIEA), and medial artery. The commonest abdominal flap for breast reconstruction is, nowadays, deep inferior epigastric artery perforator (DIEP) flap from the view of less donor site morbidity. The added inguinal nodes are fed by retrograde flow via SCIA or SIEA. In this study, as an average, 3.67 nodes can be joined to abdominal flap. However, there were 4 percent of cases without countable nodes. This result indicates that appropriate preoperative screening is needed for this procedure.
\end{abstract}

\section{Introduction}

Vascularized lymph node transfer (VLNT) is known to be an effective way of treating lymphedema. Several lymph node sites have been reported as suitable donor sites [1], with the inguinal lymph nodes being one of the major donor sites for upper extremity lymphedema treatment. These nodes enable simultaneous VLNT and breast reconstruction by transferring an abdominal flap containing the inguinal lymph nodes. Recent reports suggest that to avoid donor site iatrogenic lymphedema, superficial inguinal nodes superior to the saphenous junction are suitable $[2,3]$. Our study aimed to identify how many nodes could be included in the abdominal flap.

\section{Methods}

We reviewed 96 multi detector computed tomography (MDCT) images

*Correspondence: Kenta Tanakura, MD

The Cancer Institute Hospital, Japanese Foundation for Cancer Research, Koto, Tokyo, Japan. E-mail: kenta.tanakura@jfcr.or.jp

This work was presented in the 5th World Symposium for Lymphedema Surgery, Linkou, Taiwan, held on 27-29 Apr, 2016.

Received: Apr. 30, 2017; Accepted: Sep. 15, 2017; Published: Nov. 7, 2017

International Microsurgery Journal. 2017;1(3):1

DOI: $10.24983 /$ scitemed.imj.2017.00041

Copyright $₫ 2017$ The Author(s). This is an open-access article distributed under the terms of the Creative Commons Attribution 4.0 International License (CC-BY). taken perioperatively from the left and right sides of the abdomen of 48 cases while preparing for deep inferior epigastric perforator (DIEP) flap breast reconstruction surgery between 2011 and 2014. All the cases were females. We counted the number of superficial inguinal lymph nodes superior to the saphenofemoral junction. These nodes were fed by the superficial inferior epigastric artery (SIEA) and the superficial circumflex iliac artery (SCIA), and could be attached to the abdominal flap.

\section{Results}

The number of lymph nodes ranged between 0 and 10 (Figure 1), with 3 in 25 abdominal side images (26\%), and 4 in 18 side images (19\%). The average number of lymph nodes in a single side image was 3.67. No significant differences existed between the left and right abdominal side images. There were 4 cases with no countable lymph nodes.

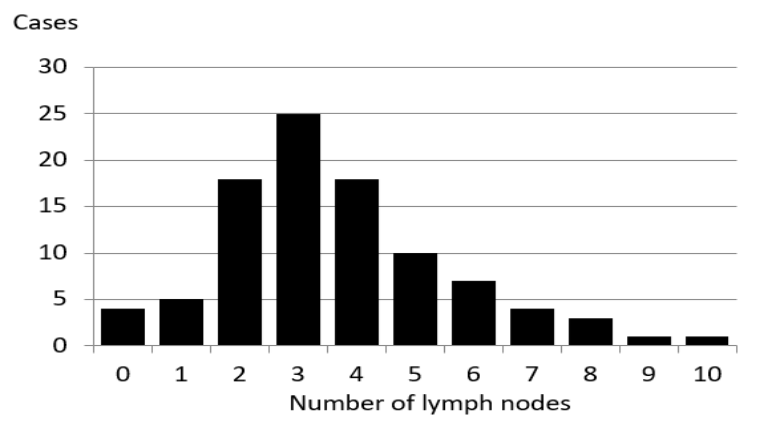

Figure 1. Number of superficial inguinal lymph nodes in superior part. 


\section{Case Report}

A 54-year-old woman had lymphedema in the left upper extremity caused by total mastectomy with axillary lymph node dissection for breast cancer; she had undergone surgery, chemotherapy and radiation therapy, one year earlier. There was no improvement in the symptoms following conservative treatment with compression therapies. There was significant fibrosis in the upper extremity and shallow pitting edema was observed, indicating a reversible state of lymphedema. We defined end-to-side lymphaticovenular anastomosis of this patient's lymphedema stage as IIb in ILS scale.

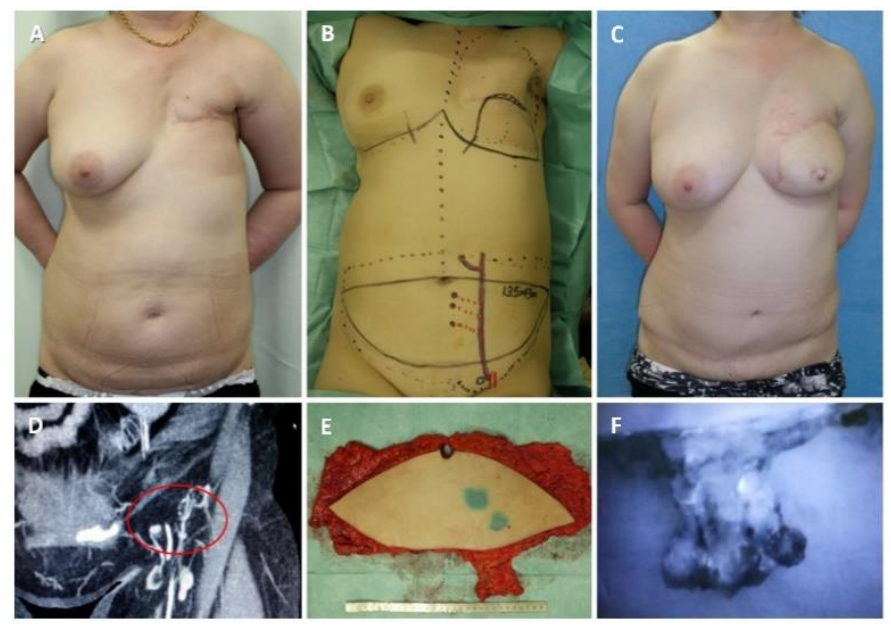

Figure 2. A 54-year-old female, after total mastectomy for her left breast cancer. Post chemotherapy and radiation. Lymphaticovenular anastomoses were performed formerly in her left dorsum of hand (A). DIEP flap with lymph nodes (LNS) along SIEA was transferred ( $B$ and $E$ ). We planned to include $4 \mathrm{LNs}$ in the flap according to enhanced MDCT (D). The LNs were well enhanced by intravenous injection of ICG (F). The flap was set in longitudinal position, and the LNs were inserted to axilla. Slight decrease of circumference was observed in mainly in her upper arm postoperative one year (C).

\section{Table 1. The Circumferential Change in Left Upper Extremity}

\begin{tabular}{|ccc|}
\hline \multicolumn{2}{|c|}{ Areas } & Circumference $(\mathrm{cm})$ \\
\hline & Pre-operation & Post-operation \\
\hline Axilla & 38.0 & 36.5 \\
\hline Arm & 29.0 & 30.0 \\
\hline Forearm & 27.0 & 28.0 \\
\hline Wrist & 17.0 & 17.5 \\
\hline Hand & 18.0 & 18.5 \\
\hline
\end{tabular}

As the surgical therapy for the lymphedema, two were performed in the left dorsum of her hand (Figure 2A). Slight improvement in her hand was felt as decrease in swell, but insufficient. One year later, DIEP flap with lymph nodes along SIEA was transferred (Figure 2B and 2E). We planned to include 4 lymph nodes in the flap according to enhanced MDCT (Figure 2D). The lymph nodes were well enhanced by intravenous injection of indocyanine green (ICG) after harvesting of the flap. We could detect at least 3 lymph nodes in the flap (Figure 2F). The flap was set in longitudinal position. The subcutaneous tissue in axilla region was too hard and thin to dissect it safely, probably due to the late effect of radiation therapy. So, a pocket for lymph node flap was made just under the axilla, and the lymph nodes were inserted to it. Slight decrease in circumference was observed mainly in her upper arm after 1-year postoperatively (Figure 2C). The circumferential change in left upper extremity was mentioned (Table 1). Donor site complication, such as newly caused lymph edema in the lower extremity or seroma in the abdomen, was not experienced. In this case, improvement of the circumference was limited in just around the axilla, probably due to insufficient subcutaneous dissection in the area.

\section{Discussion}

The lymph nodes-added abdominal flap transfer is a promising procedure, which enables simultaneous breast reconstruction and lymphedema treatment. The superficial inguinal lymph nodes are fed by the SCIA, SIEA, and the medial artery [4]. Recent studies have shown that nodes in the inferior-medial and the central areas of the saphenofemoral junction should not be selected as donor sites for VLNT, because they are the primary superficial nodes draining the lower limb [2,3]. Nodes in the superior area of the saphenofemoral junction are said to be safe, but they can be the draining nodes for the lower limb in $15 \%$ of the cases [3]. Scaglioni et al. [5] showed that the superficial inguinal lymph nodes could be divided into three subgroups: abdominal, medial thigh, and lateral thigh nodes. The dominant lymph nodes draining the leg were in the lower part of the inguinal triangle. To avoid iatrogenic lymphedema, reverse lymphatic mapping is useful to distinguish between "dangerous" nodes and nodes suited to VLNT [6]. In our case report, we couldn't use this method, because it was performed before publishing the method.

Nowadays, the workhorse flap for breast reconstruction is the DIEP flap due to less subsequent donor site morbidity. The added inguinal nodes are fed by retrograde flow via SCIA or SIEA. This study shows that, on average, 3.67 nodes can be included in the abdominal flap. The number of nodes ranged between 0 and 10 . Chen et al recently reported that the number of vascularized lymph nodes transferred correlated positively with the degree of volume reduction in rats [7]. The side with more lymph nodes should be chosen for transfer. It was worth noting that there were 4 percent of cases with no countable nodes. This result indicates that appropriate preoperative screening is recommended for this procedure.

\section{Conclusion}

We reviewed the MDCT images to show the number of lymph nodes superior to the saphenofemoral junction. In this study, on average, 3.67 nodes existed. However, there were 4 percent of cases with no countable nodes. This result indicates that appropriate preoperative screening is needed for this procedure.

\section{Keywords}

Lymphedema; vascularized lymph node transfer; deep inferior epigastric artery perforator flap; breast reconstruction; inguinal node.

\section{Article Information}

Conflict of Interest Disclosures: None Funding: None

\section{References}

1. Tourani SS, Taylor GI, Ashton MW. Vascularized lymph node transfer: a review of the current evidence. Plastic and Reconstructive Surgery 2016;137:985-993.

2. Van der ploeg IM, Kroon BB, Valdés olmos RA, Nieweg OE. Evaluation of lymphatic drainage patterns to the groin and implications for the extent of groin dissection in melanoma patients. Annals of Surgical Oncology 2009;16:2994-2999.

3. Viitanen TP, Mäki MT, Seppänen MP, Suominen EA, Saaristo AM. Donor-site lymphatic function after microvascular lymph node transfer. Plastic and Reconstructive Surgery 2012;130:1246-1253.

4. Cheng $\mathrm{MH}$, Chen SC, Henry SL, Tan BK, Lin MC, Huang JJ. Vascularized groin lymph node flap transfer for postmastectomy upper limb lymphedema: Flap anatomy, recipient sites, and outcomes. Plastic and Reconstructive Surgery 2013;131:1286-1298.

5. Scaglioni MF, Suami H. Lymphatic anatomy of the inguinal region in 


\section{Rapid Communication}

aid of vascularized lymph node flap harvesting. Journal of Plastic, Reconstructive and Aesthetic Surgery 2015;68:419-427.

6. Dayan JH, Dayan E, Smith ML. Reverse lymphatic mapping: a new technique for maximizing safety in vascularized lymph node transfer. Plastic and Reconstructive Surgery 2015;135:277-285.
7. Nguyen DH, Chou PY, Hsieh YH, et al. Quantity of lymph nodes correlates with improvement in lymphatic drainage in treatment of hind limb lymphedema with lymph node flap transfer in rats. Microsurgery 2016;36:239-245. 